\title{
IS COOPERATION BETWEEN EASTERN AND WESTERN MEDICINE POSSIBLE IN BULGARIA?
}

\author{
Paraskeva Mancheva \\ University Centre for Eastern Medicine, Training and Research Sector of Rehabilitation, \\ Medical University of Varna
}

\begin{abstract}
INTRODUCTION: Bulgarian patients are increasingly looking for Eastern (traditional) medicine (EM) as an addition to their basic treatment. In order to avoid a conflict between the methods of Eastern and Western medicine (WM) in Bulgarian conditions, it is necessary to determine the possibilities for their joint application.

AIM: The purpose of the article is to explore and present the opportunities for cooperation between Eastern and Western medicine in Bulgarian conditions.

MATERIALS AND METHODS: A review and content analysis of bibliographic sources, relevant scientific articles and Bulgarian legislation normative acts were performed.

RESULTS AND DISCUSSION: WM affects the symptom, focuses the treatment on individual organs and systems providing fast results, but the treatment has its side effects. Eastern medicine treats the individual in his entirety, mostly without side effects, cures the root cause, but the results are obtained through a prolonged treatment. Disadvantages of both types of medicine: alternative medicine is unable to deal with diseases that arise in people from the West due to contaminated environment, noise factors, impact of chemical agents, etc.; in WM there are cases of sustained and irreversible health effects caused by iatrogenesis. In recent decades in Europe and around the world, the practicing of EM techniques has been implemented as part of the prophylaxis and as a complementary therapy next to drug treatment.

CONCLUSION: Bulgarian legislation regulates the training and practicing of some methods that are part of alternative medicine („unconventional methods“). The EM methods have their place in the prophylaxis and treatment of the Bulgarian patient. A cooperation between Eastern and Western medicine is possible in Bulgarian conditions.
\end{abstract}

Keywords: Eastern medicine, unconventional methods, complementary medicine, Bulgaria

Address for correspondence:

Assoc. Prof. Paraskeva Mancheva, MD, PhD

Medical University of Varna

University Centre for Eastern Medicine

84 Tzar Osvoboditel Blvd

9002 Varna, Bulgaria

e-mail:p_mancheva@abv.bg

Received: October 11, 2018

Accepted: October 30, 2018

\section{INTRODUCTION}

The World Health Organization (WHO) defines Eastern medicine (EM) (traditional medicine, complementary medicine) as "the total amount of knowledge, skills and practices about health based on theories, beliefs and experiences coming from different cultures, whether they can be explained or not, as well as the prevention, diagnosis, improvement or 
treatment of physical and mental illnesses" (1). Traditional practices include Ayurveda, Traditional Chinese Medicine, Acupuncture, Marma therapy and others. The wide variety of EM`s methods makes it difficult to harmonize terminology and classify it $(2$, $3,4)$.

EM dates back to more than 5000 years ago. During these millennia, it has been actively evolving in the East, and in the last century in the West. Traditional Chinese Medicine, for example, is so complex and diverse that it is studied in public universities as a 5- to 7-years course of study. The future practitioners who are studying in medical schools in Europe and the world do not receive information about Eastern medicine and its methods of therapeutic influence.

Discussions on the issue of cooperation between Eastern and Western medicine (WM) in Bulgaria have just recently started. They are mainly aimed at complementing conventional treatment with the opportunities offered by EM, and not focused on the use of EM on its own. The negative reception of EM from patients and, especially, doctors is, on one hand, due to the accumulated experience so far in our country, culture, customs and traditions of the Bulgarian patient and, on the other side, the legal regulation of the issue. The normative framework of unconventional methods (UMs) is contained in the Health Act (HA), and their application is regulated through Manure Decree No. 7/01.03.2005 (5,6). In Article 166 of the HA there are seven UMs listed, and the methods that are mentioned in the law are forbidden to use. Similarly, the normative regulation of UMs (and in particular the methods of Eastern medicine) is deficient and imperfect in terms of the powers of those who can be trained and practice.

Despite these seemingly insurmountable obstacles, the Bulgarian patient's interest in EM and unconventional methods has been growing. The doctors - general practitioners and practitioners with narrow specializations - are increasingly complementing the therapy of their patients with acupuncture, acupressure, homeopathy, phytotherapy, diets, healing starvation and others. For this reason, we believe it is necessary to consider the common ground and the differences between these two worlds of medicine in order to explore the possibilities for cooperation between them.

\section{AIM}

The aim of this article is to explore and present the differences and the similarities between the practices of Traditional Chinese and Western medicine in Bulgarian conditions in order to answer the question of cooperation between them.

\section{MATERIALS AND METHODS}

A review and content analysis of bibliographic descriptions, relevant articles and normative acts of the Bulgarian legislation were performed.

\section{RESULTS AND DISCUSSION}

The Scientific Advisory Council of the European Academies considers that there should be equality in medical assessments: "There cannot exist two types of medicine - conventional and alternative. There is only medicine that has been adequately tested and medicine that is not" $(7,8)$. Conventional (Western) medicine is "evidence-based medicine” (EBM)" and is more easily susceptible to double-blind testing (placebo). In Traditional (Eastern) Medicine there is no placebo (open trial). WM is a science of the disease based on EBM. EM is characterized by the holistic approach and the understanding of the human as a unified system based on empirical experience. All phenomena of the outside world, incl. man and nature, are interpreted by EM as an interaction between Yin and Yang. The origin and development of the disease is perceived as a result of an imbalance between these two principles. The root cause is being sought and prophylaxis is performed.

What are the common things and differences between these two types of medicine?

Undoubtedly both types of medicine are oriented towards the human and educating him about a healthy way of life. However, despite the general consensus in the comparison of Eastern and Western medicine, there are a number of significant differences. EM is nature-centered, stable and conservative (9).

The understanding of its essence is associated with contemplation and with the application of the inductive approach and the analogy in diagnostics and treatment. WM is anthropocentric, mobile, pro- 
gressively changing and evolving. It is distinguished by a materialistic perception of reality from the beginning of its development to the present and applies an analytical, empirical and experimental approach to human and environmental learning.

WM considers that the human organism is not ideal and defines the way in which it can change to perfection. EM's understanding of an individual is related to the perceptions that the human is created ideal by default and can be treated with natural methods of influence. WM works predominantly with the consequences of the disease - tumors, stones, polyps, etc. EM detects and eradicates the energy imbalance and the cause of the disease prior to the occurrence of serious consequences.

EM uses volumetric and complex diagnostics, without the need for any technical devices, based on conversation with the patient; palpation; check of tongue, iris, nails; pulse diagnostics, etc. A disease is considered to be a disturbance of the balance and deviation from the equilibrium of the Yin and Yang forces (cold and heat, etc.). EM bases itself on a massive and strictly organized theory, which allows to accurately determining the manifestation of the dis- ease. WM is considered to be „evidence-based medicine" and applies quantitative diagnosis, on the basis of which the treatment is perfectly assigned.

WM uses usually chemically synthesized drugs, while EM is bioenergetical and uses only natural products. The main differences between Eastern and Western medicine are synthesized in the table below (Table 1).

After all, what is the answer to the question: „Is a cooperation between Eastern and Western medicine possible?"

Experts from the WHO have developed a program on the integration of folk/traditional medicine into national health systems $(10,11)$.

The aim of this program is to preserve the authenticity of healing practices that have proven effectiveness and to ensure their legal application and safety. An example of such a unique union is the health care system in China. Chinese folk medicine and modern medicine exist and are applied together. The medicinal products created under an ancient Chinese prescription undergo clinical trials. After that they are certified and manufactured by pharma-

Table 1. Differences between Eastern and Western Medicine

Western
Science of the disease
Has been in existence for circa 2000 years (since the time
of Hippocrates)
Takes into account the anatomical and physiological
features of the organism
Examines the organism as separate systems and organs

Focuses on the disease and treatment

Treats symptoms

Practitioners with professional specialization

Diagnostics performed with apparatuses

Medications with side effects

The chemical impact of the medication overloads the body

Weakens the immune system

Creates conditions for the development of drug dependence

\section{Medicine}

\section{Health science}

Very ancient (has been existing for more than 5000 years)

Also takes into account the energy, the connection with the cosmos, the Earth and the socium

Examines the organism of the human as a whole, all organs and systems are interrelated

Focuses on health and prevention

Identifies and removes the root cause

Eastern Medicine specialists

Pulse, iris and tongues diagnostics

Medications of natural (plant, animal, mineral) origin with no side effects

Detoxifies the body

Restores the immune system

Overcoming drug, alcohol and nicotine dependence 
ceutical factories. Doctors are trained in Traditional Chinese Medicine at medical schools. Official Western medicine has derived positive experiences from EM: Acupuncture's treatment of the nervous system diseases has long been recognized by the WHO.

A common ground between Western and Eastern medicine could be found when we examine the practices of treating certain diseases. For example, a hot water bathtub is often used as a treatment of hypertensive crisis. In this tub the patient is soaking his feet until the blood pressure is lowered. In case of a stroke, WM practices cooling of the head and the whole organism, while EM warms up the stomach. For treatment of malignancies, the combination of conventional with unconventional methods for controlling the pain is not uncommon $(12,13)$.

The simultaneous application of conventional and unconventional methods in Bulgaria is not a sporadic phenomenon. Researches, records and data collection on Bulgarian folk medicine coincides with the scientific botanical studies in Bulgaria at the beginning of this century (14). A series of clinical cases publications reported by the team of the University Centre for Eastern Medicine at the Medical University of Varna prove the successful implementation of the methods of EM and WM in the cases of chronic disability, spinal disc herniation (cervical and lumbar), myofascial pain syndrome, carpal tunnel syndrome and many other diseases $(15,16,17)$.

Recently, a review of the European complementary and alternative medicine therapeutic methods has been published which shows again that EM and WM may share a mutual path in the future (18). These and other facts indicate that there is a practice - in our country and worldwide - of application of the EM methods as a complementary therapy to the main treatment that WM provides.

The benefits from the EM-WM cooperation have been empirically proven by many satisfied patients. Researchers try to prove that through mixedmethod approaches, through measuring patient-reported outcomes like health-related quality of life and through successful case study reports $(19,20)$.

\section{CONCLUSION}

Undoubtedly, the complete replacement of WM with EM in the Western world is impossible and un- necessary. This is because, for millennia, each of them has emerged in a certain territory and has imposed its influence over these areas under different cultural conditions, customs and traditions. Definitely, cooperation between EM and WM is possible despite some shortcomings: EM's inability to deal with diseases that occur to people in the West due to contaminated environment, noise, impact of chemical agents, etc.; WM's sustained and irreversible health effects caused by iatrogenesis. The patients' needs and the public health responsibilities of the state and the society towards improving population and individual health call for mutual use of the medical methods of both worlds - the East and the West.

\section{REFERENCES}

1. Traditional Medicine: Definitions. World Health Organization. Available from: http://www.who.int/ traditional-complementary-integrative-medicine/ en/. 11.10.2018.

2. Council of Europe. Legislation and administrative regulations on the use by licensed health service personnel of non - conventional methods of diagnosis and treatment of Illness, Strasbourg. 1984.

3. NCCAM, National Center for Complementary and Alternative Medicine. Available from: http://nccam.nih.gov/news/camstats/2007/camsurvey_fs1. htm. 11.10.2018.

4. WHO Traditional Medicine Strategy 2002-2005. Available from: http://whqlibdoc.who.int/hq/2002/ WHO_EDM_TRM_2002.1.pdf. 11.10.2018.

5. State Gazette (official edition of the National Assembly). Health law. №70/10.08.2004 in effect from 01.01.2005/ recent changes SG №18 /27.02.2018 [in Bulgarian].

6. Ordinance №7/01.03.2005 [in Bulgarien].

7. Angell M, Kassirer J. Alternative Medicine - The Risks of Untested and Unregulated Remedies. N Engl J Med. 1998;(339):839-841. DOI: 10.1056/ NEJM19980917339121.

8. Grozdeva D, Dikova Ts. Gas discharge visualization - historical developments, research dynamics and innovative applications Scripta Scientifica Salutis Publicae. 2018; (4):27-33.

9. Legal Status of Traditional Medicine and Complementary/Alternative Medicine: A Worldwide Review - 2001. Available from: http://apps.who.int/ medicinedocs/en/d/Jh2943e/3.3.html.11.10.2018. 
10. European Parliament. Resolution of the EP on Non-Conventional Medicine A4-0075/97. Committee on the Environment, Public Health and Consumer Protection. Available from: http://www.europarl.europa.eu/sides. 11.10.2018.; 20.

11. WHO traditional medicine strategy: 2014-2023, Available from: http://www.who.int/medicines/ publications/traditional/trm_strategy14_23/en. 11.10.2018.

12. Menefee LA, Monti DA. Nonpharmacologic and complementary approaches to cancer pain management. J Am Osteopath Assoc. 2005;(105):15-20.

13. Ripamonti C, Dickerson ED. Strategies for the treatment of cancer pain in the new millennium. Drugs. 2001;(61):955-77.

14. Stanev St. Few familiar names from Bulgarian botany. Pensoft; 2001 [in Bulgarian].

15. Mancheva $P$, Shivachev $Y$. Unconvetionals Methods of Treatment as a Part of the Holistic Approach in the Management of the Chronic Invalidizing Disease. Health Economics and Management. 2017;2(64):33-35.

16. Shivachev Y, Grozdeva D, Mancheva P, Bogomilova St, Nenova G, Kostadinova T. Multidisciplinary cooperation between complementary and conventional medicine with patient suffering from myofascial pain syndrome. Journal of IMAB. 2018;24(3):2125-2128.

17. Shivachev Y, Grozdeva D, Mancheva P. Complementary versus Conventional medicine in Carpal tunnel syndrome? A case report. Scripta Scientifica Medica. 2017;49(4):67-70.

18. Kapincheva I, Vankova D. Pan-European Concept "Complementary and Alternative Medicine" (CAM) - History, Content and Public Health Importance, Social Medicine. 2018;(2):18-22.

19. Vankova D. Community-centered research in Bulgaria, a mixed-methods approach to health-related quality of life, European Journal of Public Health, 8th European Public Health Conference: Proceedings. 2015;25(3):291.

20. Vankova D. Conceptual and Methodological Approaches to Quality of Life- a public health perspective, Scripta Scientifica Salutis Publicae. 2015;1(2):7-13. 